\section{Dental management of the tropical disease human African trypanosomiasis: an unusual case of pseudobulbar palsy}

L. Frost ${ }^{1}$

IN BRIEF
- Explains the dental and neurological
sequelae of sleeping sickness.
- Outlines the impact of migration medicine
on dentistry.
- Provides revision of neuroanatomy and
cranial nerve examination.
Discusses the xerostomia side-effect of
some medications.
- Highlights the importance of a multi-
disciplinary management approach to
treating dental patients.

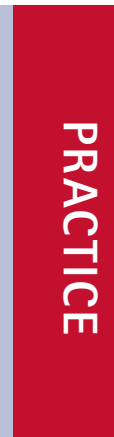

Human African trypanosomiasis (sleeping sickness) is a parasitic tropical disease endemic to sub-Saharan Africa. Due to migration and holiday travel patterns cases are increasing in the United Kingdom. The neurological sequelae have dental management implications both directly from the consequent physical disability and indirectly from the oral side-effects of the medications used to manage symptoms. Changes in disease demographics require the dental profession to increase its awareness of migration medicine and the appropriate dental management of such diseases.

\section{EPIDEMIOLOGY}

Migration to the UK from Africa is on the increase, including the sub-Saharan region where sleeping sickness is prevalent. In 2009 the UK issued 396,690 visas for African nationals, accounting for $25 \%$ of the total migrant population into the UK. ${ }^{1}$ Recent estimates by the World Health Organisation $^{2,3}$ indicate that over 60 million people living in some 250 locations are at risk of contracting Human African trypanosomiasis, and there are about 300,000 new cases each year. The disease has been recorded as naturally occurring in 36 countries, all in sub-Saharan Africa. It is endemic in southeast Uganda and Western Kenya and kills more than 40,000 Africans a year. ${ }^{2,3}$ Since 2005 there have been 11 imported cases in Europe, two in the UK, ${ }^{4}$ and a total of 16 cases in the UK since $1998 .^{5}$

\section{PATHOGENESIS}

Human African trypanosomiasis is a vector-borne parasitic disease that has been present in Africa since at least the fourteenth century. The parasites concerned are protozoa belonging to the Trypanosoma

'Dental Officer, Solent Healthcare Dental Service, Dental Department, Bitterne Health Centre, Commercial Street, Southampton, Hampshire, S018 6BT

Correspondence to: Miss Laura Frost

Email: laura.frost@solent.nhs.uk

\section{Refereed Paper}

Accepted 30 September 2010

DOI: 10.1038/sj.bdj.2010.1181

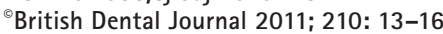

genus, transmitted to humans via the bites of tsetse flies, which acquire their infection from human beings or animals (Fig. 1). ${ }^{6}$ The causative agent and vector were not identified until 1902-1903 by Sir David Bruce, and the differentiation between the sub-species of the protozoa was not made until 1910. Tsetse flies are located in subSaharan Africa and only certain species transmit the disease.

Human African trypanosomiasis takes two forms, depending on the parasite involved:

1. Trypanosoma brucei gambiense is found in west and central Africa. It accounts for more than 90\% of sleeping sickness cases and causes a chronic infection. Time between infection and clinical symptoms can be months or even years

2. Trypanosoma brucei rhodesiense accounts for $10 \%$ of sleeping sickness cases. It is found in eastern and southern Africa and causes an acute infection. First signs and symptoms are seen a few weeks after infection and the disease develops rapidly, invading the central nervous system. ${ }^{2}$

\section{SYMPTOMS AND DISEASE CONSEQUENCES}

The first stage (haemolymphatic phase) is characterised by fever, headaches, joint pains and itching. The second stage (neurological phase) begins when the parasite crosses the blood-brain barrier and invades the central nervous system. In general this encephalitic stage is when the signs and symptoms of the disease appear: confusion, sensory disturbances, poor coordination and disturbance of the sleep cycle.

Treatment of the second stage is with arsenic preparations, such as Melasaprol, that cross the blood-brain barrier. The medication can have a high level of toxicity, sometimes causing a reactive encephalopathy (encephalopathic syndrome) and prove lethal. However, without treatment sleeping sickness is invariably fatal too as it progresses with mental deterioration leading to coma and death; if the individual survives, damage caused in the neurological phase can be irreversible.

\section{CASE REPORT}

A 40-year-old Caucasian British-born gentleman was referred by medical staff at a brain rehabilitation care home to the community dental service, due to his complex neurological condition, for a routine examination. The rarity of sleeping sickness in the UK prompted a letter to his consultant of tropical medicine who kindly provided further background information, summarised as follows from the journal article by Checkley et al. ${ }^{7}$ The man had been travelling for 2.5 years in Malawi, Mozambique, Namibia and South Africa, where he presented to a hospital with a four-month history of progressive fatigue, sleeplessness, severe headache and pyrexia. 
Doctors in South Africa diagnosed him with Trypanosoma brucei rhodesiense infection and treatment consisted of a suramin, melarsoprol and prednisolone regime. The patient was transferred to the Hospital for Tropical Diseases in London where he initially made a full recovery but subsequently relapsed and developed melarsoprol-associated encephalopathy requiring admission to intensive care for life-support intervention. Subsequent recovery was initially characterised by locked-in syndrome that gradually improved resulting in a left pyramidal syndrome and cognitive impairment with spastic quadriparesis, pseudobulbar palsy, opthalmoplegia and abulia. The patient was transferred to a residential brain rehabilitation unit for 24-hour medical and nursing care and referred to the community dental service for continuing dental care due to his complex medical condition and physical disability.

Before the sleeping sickness infection the gentleman had been fit and healthy, but at the initial dental examination extensive neurological damage was observed, restricting him indefinitely to a motorised wheelchair.

Pseudobulbar palsy is bilateral impairment in function of the brainstem upper motor nuclei and therefore weakness or paralysis of muscles supplied by the lower cranial nerves: glossopharyngeal, vagus, accessory and hypoglossal. In this patient it resulted in paralysis of the muscles innervated by the medulla, causing dysphagia, dysphonia, myoclonus of lips, tongue, soft palate and vocal cords, and restriction of neck movements (sternocleidomastoid and trapezius muscles). The patient also had some upper motor neuron involvement of the intercostal muscles resulting in poor respiratory reserve causing intermittent 'panting'. Previously, during initial medical intervention, the patient had had a tracheostomy but there was not believed to be any consequent tracheal stenosis. In line with the Mental Capacity Act 2005 the patient had capacity to consent.

Dental examination was undertaken while the patient remained in their own wheelchair. Extra-orally the patient's speech was slow and slurred. The findings of the complete cranial nerve examination are summarised in Table 1 . The right arm had movement but the left hand and wrist were in a thermoplastic dorsal splint.

\begin{tabular}{|c|c|c|}
\hline Cranial nerve & $\begin{array}{l}\text { Intact function: } \\
\text { yes, no or reduced }\end{array}$ & Findings \\
\hline 1. Olfactory & YES & Sense of smell intact \\
\hline 2. Optic & YES & Visual acuity normal \\
\hline 3. Oculomotor & NO & $\begin{array}{l}\text { Diplopia, pupils non-reactive to light (directly or indirectly) } \\
\text { fixed non-dilated }\end{array}$ \\
\hline 4. Trochlear & NO & Diplopia \\
\hline 5. Trigeminal & YES & Facial sensation intact \\
\hline 6. Abducens & NO & Diplopia, paralysis of eye abduction \\
\hline 7. Facial & REDUCED & $\begin{array}{l}\text { Able to raise eyebrows, puff out cheeks and smile; } \\
\text { however myoclonus of eyelids and lips }\end{array}$ \\
\hline 8. Vestibulocochlear & YES & $\begin{array}{l}\text { Unable to assess balance as wheelchair bound but } \\
\text { no dizziness. Hearing normal. }\end{array}$ \\
\hline 9. Glossopharyngeal & NO & No gag reflex \\
\hline 10. Vagus & NO & Hoarse voice, no gag reflex \\
\hline 11. Accessory & REDUCED & Able to turn neck to both sides but not shrug left shoulder \\
\hline 12. Hypoglossal & REDUCED & Tongue: no deviation but myoclonus \\
\hline
\end{tabular}

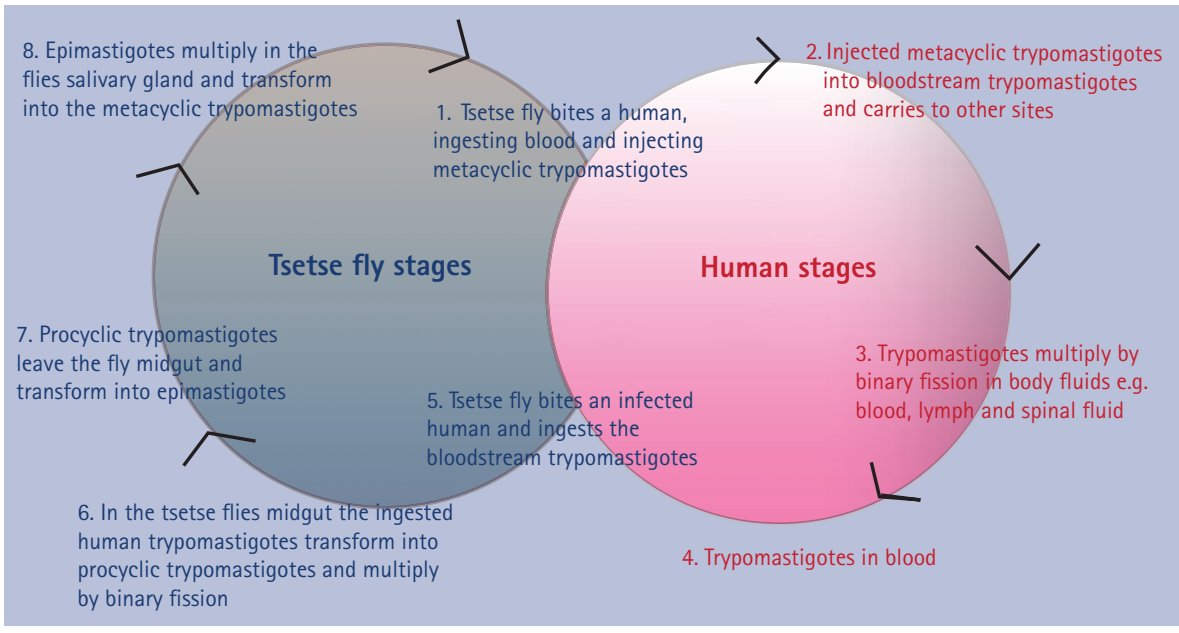

Fig. 1 Life cycle of the Trypanosoma brucei parasites

Pseudobulbar palsy is documented as causing an increased jaw jerk reflex but it was not tested in this patient to minimise their anxiety of dental treatment.

Intra-oral examination was limited to short bursts to enable the patient to maintain a normal breathing pattern. The soft tissues were healthy but there was some 'slough' of dead gingivae cells present labial to the upper incisors (Fig. 2), due to poor oral hygiene, easily removed with a cotton wool roll to reveal healthy gingivae underneath. The mouth was slightly moist with stringy saliva. Oral hygiene was very poor with calculus on all surfaces, recording a Basic Periodontal Examination score of 2 in all sextants, causing generalised marginal gingivitis (Figs 2 and 3). Caries was visible in the upper right first molar and upper right central and lateral incisors. Bitewing radiographs (Fig. 4) taken to assess caries showed extensive decay in the upper right first molar and upper left third molar, with subgingival calculus evident generally.

A swallow assessment by ear, nose and throat specialists had shown impairment at the pharyngeal stage with no clearing of residue in the pharynx, creating a high risk of aspiration. The patient was therefore nil by mouth, receiving all food and fluids via a percutaneous endoscopic gastrostomy (PEG) and not allowed chewing gum or any preparations to lubricate their mouth to minimise saliva production and reduce aspiration risk. Mouthwash was permitted 


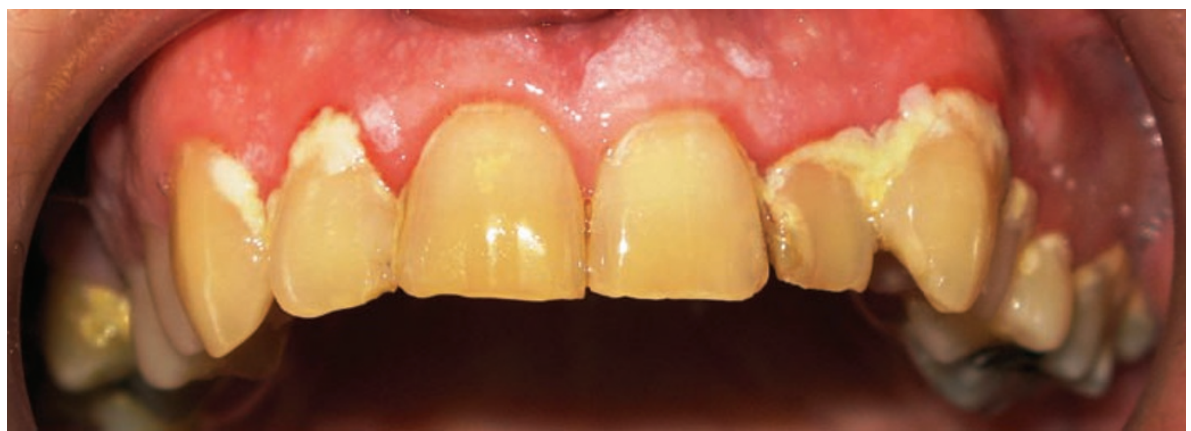

Fig. 2 Poor oral hygiene upper labial and buccal segments

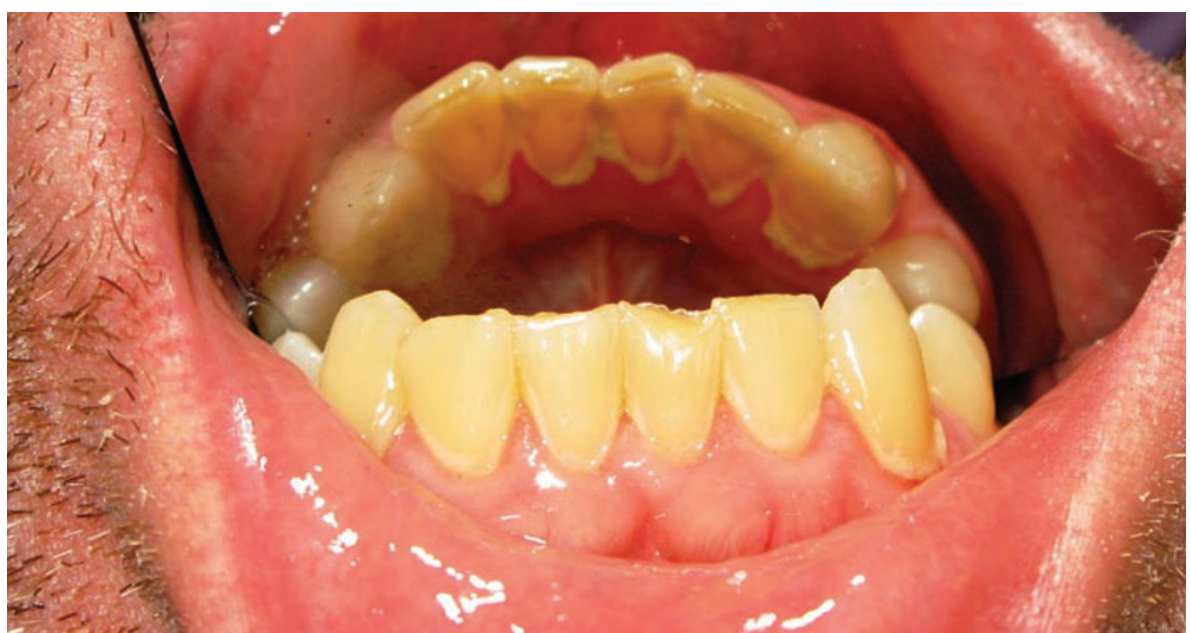

Fig. 3 Initial clinical findings lower anterior segment

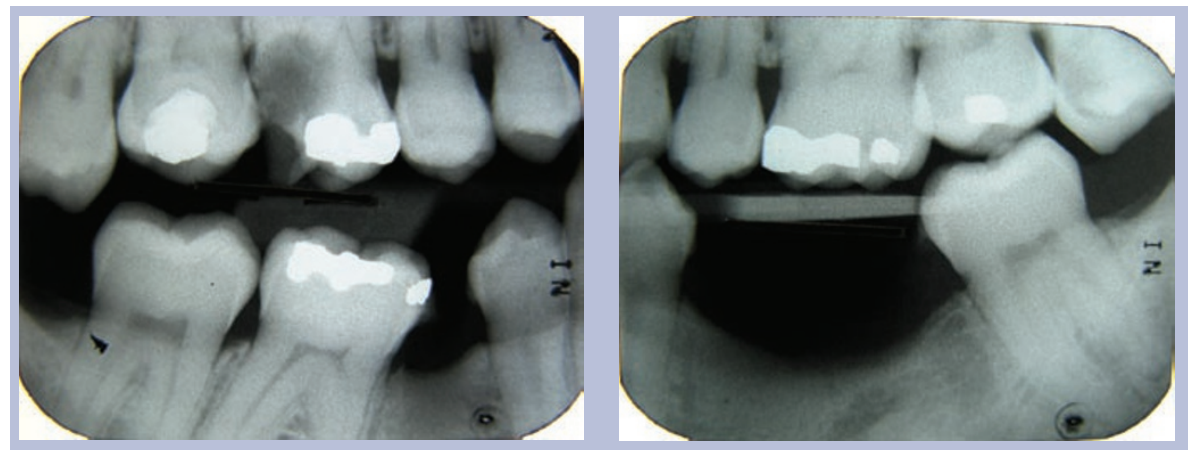

Fig. 4 Bitewing radiographs showing caries in the upper right first molar and upper left third molar

as the patient could control his facial muscles to expel the liquid.

The list of medication was extensive with the following known to cause xerostomia: hyoscine (prescribed for bladder spasms), tizanidine, solifenacin succinate and amitriptyline.

Following initial clinical assessment and discussion with the patient about the dental treatment required, it was decided to take a multi-disciplinary management approach. To obtain further information about the disease and any specific considerations before dental treatment, letters were sent to his consultant in rehabilitation and brain injuries, a professor at the London Hospital of Tropical Medicine of further caries and periodontal disease was paramount; to reduce the xerostomia risk the dose of amitriptyline was reduced gradually then stopped by his doctor. The other anti-muscarinic medications could not be stopped for important medical reasons. Corsodyl gel was prescribed for use at a different time to Duraphat 5,000 ppm Ftoothpaste twice daily.

To achieve a stable healthy mouth, oral hygiene, restorations and extractions were required. Composite restorations were able to be completed on the upper right lateral and central incisors under local anaesthetic in our dental surgery with the patient in his own wheelchair. The high speed handpiece was used minimally with reduced water, to avoid inhalational aspiration, and short bursts of treatment so as to avoid pulpal damage, with high volume aspiration used throughout. Unfortunately the use of a rubber dam was not permitted due to the airway difficulties.

A referral was made to the hospital oral surgery department for extraction of the upper right first molar and upper left third molar under local anaesthetic because they were possibly surgical extractions combined with difficult access, a high risk of aspiration and poor respiratory reserve meaning treatment was limited to a short duration. Access was difficult in the community dental clinic because no suitable hoist or wheelchair tipper was available; it was therefore felt more appropriate to refer him to the hospital dental service where more appropriate equipment was available.

To maintain a good level of periodontal health regular hand-scaling, with continuous high volume aspiration, was completed every three months or more frequently as required. Oral hygiene advice was given to support the patient's daily toothbrushing as they were finding it problematic due to wearing hand/arm splints.

A long-term dental care plan was essential due to the permanent nature of the neurological problems. Regular prevention advice to the patient and brain rehabilitation unit staff has helped to stabilise his periodontal condition and achieved a reduction in caries risk using high fluoride adjuncts. The ongoing challenges remain but the outlook is more optimistic in that one barrier to this gentleman living as normal a life as possible after a tragic lifechanging illness has been removed. 


\section{DISCUSSION}

The World Health Organisation (WHO) describes sleeping sickness as the deadliest disease in the world, classing it as a neglected tropical disease ${ }^{8}$. The term neglected refers to the fact that it exists entirely in the poorest communities, having been eradicated elsewhere and often forgotten by the rest of the world. Over 1 billion people (one sixth of the world's population) are affected by one or more neglected tropical diseases (NTDs). The group of 15 different NTDs monitored by the WHO includes leprosy, dengue haemorrhagic fever and trachoma. ${ }^{8}$

Population migration patterns have a direct correlation with the spread of infectious diseases, with many conditions increasingly presenting in countries in which they would not naturally reside. Sixteen cases of sleeping sickness in the UK since 1998 suggest it is not content with being 'neglected' but is here to stay and advancing its borders. ${ }^{5}$

Knowledge of how tropical conditions can affect patients systemically, and more specifically in the head and neck region, is useful to ensure appropriate management of dental treatment. In the recent article by Professor St John Crean, ${ }^{8}$ a proposal was made for an increase in the dental undergraduate syllabus content of migration medicine. The author agrees a greater knowledge of tropical medicine would benefit dental professionals in understanding the implications for dental management of patients suffering from these conditions.

Antimuscarinic medications, such as hyoscine and solifenacin, block parasympathetic muscarinic receptors. In addition they also block the few cholinergic sympathetic neurons which are located in the salivary glands causing xerostomia. Tricyclic antidepressants, such as amitriptyline, also have antimuscarinic side-effects. The xerostomia side-effect of medication is an important consideration before dental treatment planning, as highlighted by this case report, to tailor the oral health advice and consider the prescription of high fluoride toothpastes/mouthwashes.

Working closely with medical colleagues is good practice and essential to ensure a high standard of patient management both short- and long-term. It has the benefit of easily collating additional medical information such as medication lists and details of unusual conditions. Collaboration between the different medical and dental teams enables treatment and appropriate medication changes to be facilitated more quickly. Early involvement with medical colleagues in the patient's management means that any changes to medications and treatments take into consideration the oral side-effects.

\section{CONCLUSION}

Migration medicine poses a new challenge for dentistry in the United Kingdom. The everchanging demographic of our patient base requires the dental profession to adapt to the increasing variety of diseases we encounter.

Pseudobulbar palsy can be as a consequence of common medical conditions such as strokes, motor neurone disease and multiple sclerosis. The tropical disease sleeping sickness described in this case report highlights the importance of also being aware of how even rare medical problems can cause complex neurological sequelae and impact on dental management.

1. Home Office. Control of Immigration:Statistics, United Kingdom, 2009. Home Office Statistical Bulletin: 2010.

2. World Health Organisation Media Centre. African trypanosomiasis or sleeping sickness. Fact sheet 259: 2010.

3. World Health Organisation Media Centre. African trypanosomiasis or sleeping sickness. Fact sheet 259: 2001

4. Gautret $P$, Clerinx J, Caumes E et al. Imported human African trypanosomiasis in Europe, 2005-2009. Euro Surveill 2009; 14: pii: 19327.

5. Moore D A, Edwards M, Escombe R et al. African trypanosomiasis in travellers returning to the United Kingdom. Emerg Infect Dis 2002; 8: 74-76.

6. Centre for Disease Control and Prevention: Division of Parasitic Diseases. http://www.dpd.cdc.gov/dpdx/ HTML/ImageLibrary/TrypanosomiasisAfrican_il.htm

7. Checkley A M, Pepin J, Gibson W C, Taylor M N, Jager H R, Mabey D C. Human African trypanosomiasis: diagnosis, relapse and survival after severe melarsoprol-induced encephalopathy. Trans R Soc Trop Med Hyg 2007; 101: 523-526.

8. World Health Organisation: Control of neglected tropical diseases is feasible. http://www.who.int/ neglected_diseases/en/. Accessed 04 January 2011.

9. Crean S J. Migration medicine in dentistry: educational challenges for the future? Faculty Dental Journal 2010; 1: 26-31. 